\title{
SPECTROMICROSCOPIES ÉLECTRONIQUES: SONDER LES PROPRIÉTÉS OPTIQUES DE NANOMATÉRIAUX AVEC DES ÉLECTRONS RAPIDES
}

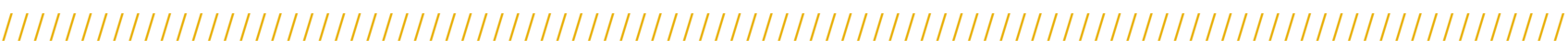

Mathieu KOCIAK ${ }^{1, *}$, Luiz Henrique GALVAO TIZEI ${ }^{1}$, Sophie MEURET' ${ }^{2}$, Hugo LOURENÇO-MARTINS ${ }^{1}$, Odile STEPHAN ${ }^{1}$

${ }^{1}$ Laboratoire de Physique des Solides, Université Paris Sud, Bâtiment 510, UMR CNRS 8502, Orsay 91400, France

${ }^{2}$ CEMES-CNRS, Université de Toulouse, 29 rue Jeanne Marvig, 31055 Toulouse, France

*mathieu.kociak@universite-paris-saclay.fr

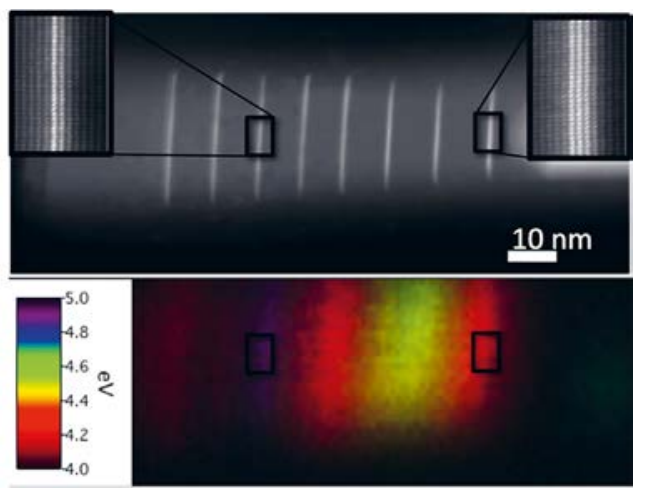

Récemment, les microscopies électroniques à balayage et/ou en transmission se sont imposées comme des techniques de choix pour étudier les phénomènes optiques avec des résolutions spatiales bien meilleures que la limite de diffraction optique. Les spectroscopies de perte et de gain d'énergie, ainsi que la cathodoluminescence permettent ainsi d'étudier à une échelle pertinente des excitations comme les plasmons de surface dans les nanoparticules métalliques ou les excitons dans des structures confinées quantiquement.

https://doi.org/10.1051/photon/202010239

Article publié en accès libre sous les conditions définies par la licence Creative Commons Attribution License CC-BY (http://creativecommons.org/licenses/by/4.0), qui autorise sans restrictions l'utilisation, la diffusion, et la reproduction sur quelque support que ce soit, sous réserve de citation correcte de la publication originale.

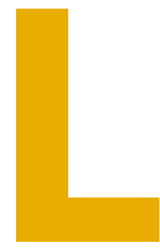

a microscopie électronique - en transmission (TEM) et/ou à balayage (S(T)EM - est communément utilisée dans un grand nombre de domaines scientifiques. On peut citer parmi une myriade d'autres applications, les techniques d'imageries et spectroscopies à la résolution atomique, dont lutilité dans le domaine des matériaux n'est plus à démontrer ou la cryomicroscopie pour l'imagerie biologique.

Une propriété moins connue de ces microscopes est qu'ils permettent de remonter aux propriétés optiques d'une large variété de matériaux à des échelles nanométriques [1], c'est-àdire à des échelles très petites devant la longueur d'onde des phénomènes optiques. La très haute résolution spatiale de ces microsopes est liée à la longueur d'onde des électrons utilisés pour éclairer l'échantillon: del'ordre du pm pour les (S)TEM ou de quelques dizaines de pm pour les SEM, permettant d'atteindre des résolutions plus petites que l'angström. La possibilité de mesurer des propriétés optiques à l'aide d'électrons peut paraitre plus contre-intuitive. Il existe pourtant des spectroscopies électroniques qui permettent de remonter aux priorités optiques (voir encart). Il s'agit tout d'abord de la cathodoluminescence (CL) [2], que nous connaissons tous ne serait-ce que de par son utilisation dans les téléviseurs du siècle dernier. La CL consiste en un mécanisme d'émission delumière suite à l'interaction d'un électron rapide avec la matière. Il y a également la spectroscopie de perte d'énergie électronique (EELS) [1], une technique surtout utilisée par le passé comme spectroscopie pour sonder les propriétés chimiques et électroniques. Il s'agit dans ce cas d’analyser l'énergie perdue par un électron rapide du fait de son interaction $\bullet \bullet$ 


\section{PRINCIPE DES PRINCIPALES SPECTROSCOPIES OPTIQUES UTILISANT DES ÉLECTRONS RAPIDES}

Le principe des spectroscopies à base d'électrons rapides pour la nanooptique est résumé dans la figure 2. La spectroscopie de perte d'énergie des électrons (EELS) repose sur l'échange d'un photon virtuel entre un électron rapide ( la moitié de la vitesse de la lumière, $\sim 100 \mathrm{keV}$ ) et un objet d'intérêt, ce qui entraîne un ralentissement de l'électron incident. L'énergie perdue par l'électron est directement liée aux excitations induites dans l'objet d'intérêt, allant des phonons aux excitations du noyau atomique. Dans le domaine optique, l'EELS fournit à l'échelle nanométrique une mesure analogue à l'extinction optique - c'est-à-dire qu'un spectre EELS est très proche d'un spectre d'absorption optique, pour des particules de très petites tailles (inférieures à quelques dizaines de nanomètres) [3]. L'énergie transmise par l'électron à l'objet d'intérêt peut être réémise sous forme de lumière, soit par diffusion de Rayleigh, soit par luminescence. La spectroscopie de cette émission de lumière est appelée cathodoluminescence (CL) [2]. Dans le premier cas, la CL est un pendant nanométrique de la diffusion optique, alors que dans le second, c'est en termes de photoluminescence nanométrique qu'il faut penser. Si un échantillon est optiquement excité par injection de lumière (Fig. 2b), un électron peut être accéléré et ainsi gagner de l'énergie au lieu d'en perdre [1]. On parle alors de spectroscopie de gain d'énergie (EEGS). De manière plus précise, l'injection de lumière induit des processus où l'électron absorbe ou émet des photons (via une nanoparticule) de manière stimulée. On parle alors de spectroscopie de perte/gain d'énergie électronique stimulés.
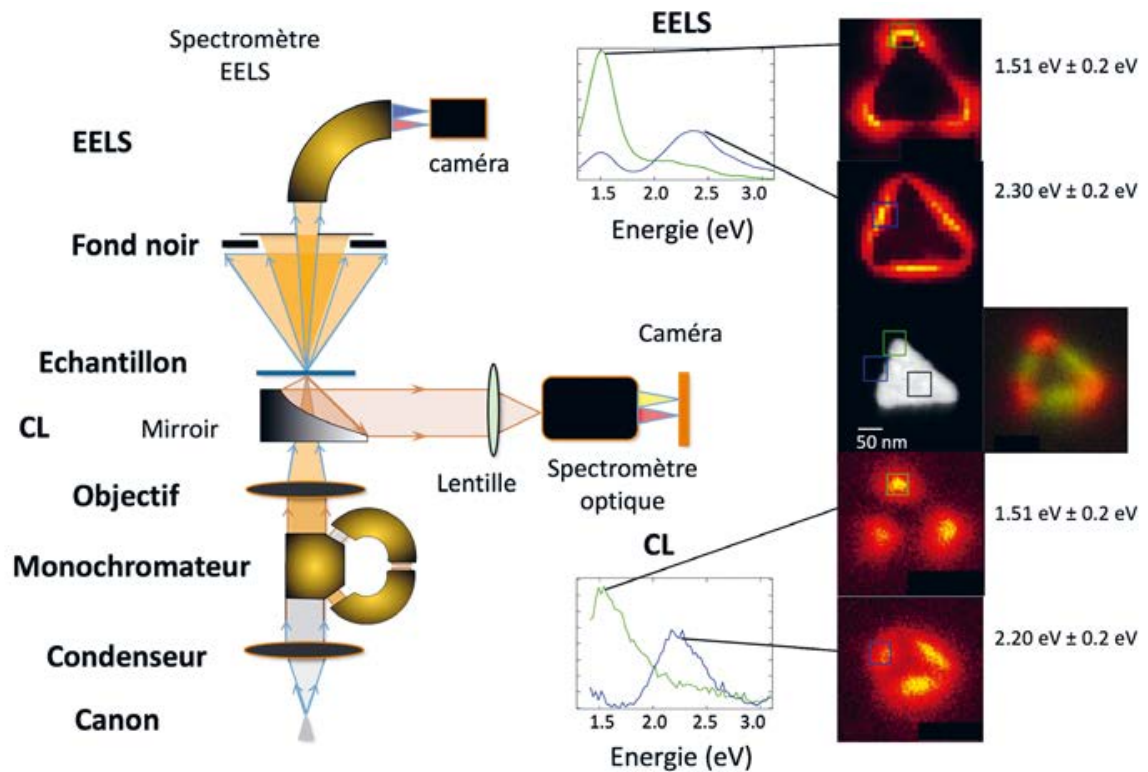

Figure 1.

Principe de fonctionnement d'un microscope électronique dédié à la spectromicroscopie pour l'optique. avec la matière. Ces deux spectroscopies sont connues depuis plus d'un demi-siècle. Cependant, c'est au cours des vingt dernières années que des ruptures technologiques et conceptuelles ont permis à ces deux techniques de devenir des outils privilégiés pour l'étude des phénomènes optiques à l'échelle nanométrique. Plus récemment, la spectroscopie de gain d'énergie a été prédite théoriquement, puis observée avec l'avènement récent des microscopes électroniques en transmission ultra-rapides (voir article de A. Arbouet et al. dans ce numéro). Cette spectroscopie est pratiquée en détectant l'accélération des électrons rapides en interaction avec des nanoobjets eux-mêmes illuminés par un rayonnement laser (voir encart).

\section{PRINCIPES PHYSIQUE ET TECHNIQUE DES SPECTROMICROSCOPIES ÉLECTRONIQUES}

Comme le montre le schéma de la figure 1, un S(T)EM fonctionne, sur le principe, comme un microscope optique [2]. Il se compose d'une source d'électrons, de lentilles (magnétiques) pour façonner et guider le faisceau délectrons, ainsi que d'éléments dispersifs (typiquement un prisme magnétique) pour effectuer une spectroscopie de perte (ou de gain) d'énergie. Un S(T)EM peut être utilisé pour acquérir des images. Pour cela, un faisceau d'électrons est focalisé sur léchantillon d'intérêt grâce à une lentille objectif. Ce faisceau de taille souvent nanométrique pour les applications optiques est balayé sur l'échantillon. En chaque point du balayage, différents signaux scalaires peuvent être acquis afin de former une image à la fin du balayage. Ces signaux ont différentes origines physiques - par exemple, émission d'électrons secondaires (SE) ou signal de fond noir (HADF). Le premier permettant de former des images dela topographie de léchantillon, le second servant à visualiser la structure et la morphologie de l'échantillon, jusqu’à des échelles atomiques. On voit par exemple le signal de fond noir d'un nanoprisme triangulaire d'argent sur la figure 1, l'argent apparaissant plus intense que le support très peu diffusant constitué de nitrure de silicium.

De façon analogue, en chaque point de léchantillon, des informations spectrales 
peuvent être acquises. Il peut s'agir de spectres EELS ou CL (voir Fig. 1) ou EEGS. Comme on le comprend déjà sur la figure 1 , la grande force de ces techniques est de pouvoir, pixel par pixel, corréler les informations optiques (les spectres EELS/CL/EEGS) aux données structurales ou morphologiques (SE, HADF...), car les deux informations sont acquises en parallèle en chaque point du balayage. Les données spectrales acquises forment ce qu'on appelle une image spectrale (c'est pour cela que lon parle de spectromicroscopie), dans laquelle, en chaque pixel, est stocké un (ou plusieurs) spectre(s) complet(s). La visualisation et l'exploitation de ces données tri-dimensionnelles n'étant pas triviales, différentes approches sont généralement utilisées. On peut par exemple générer des cartes comportant différentes quantités physiques, comme par exemple la variation spatiale del'intensité d'une signature spectrale donnée (voir les cartes correspondantà deux résonances du nanoprisme d'argent sur la figure 1), leur position en énergie ou leur largeurà mi-hauteur. Une manière plus qualitative et visuelle est de générer des cartes en fausses couleurs (voir Fig. 1), pour lesquelles l'image spectrale n'est pas considérée comme une série de spectres, mais comme une succession d'images à des énergies différentes. On voit par exemple sur la figure 1 que lon observe des excitations à plus basse énergie (rouge) localisées sur les pointes et à plus haute énergie (verte) sur les côtés, et ces modulations se faisant à très petite échelle devant les longueurs d'onde typiques du visible. On comprend bien queles spectromicroscopies permettent effectivement de mesurer des phénomènes nanooptiques. Mais comment interpréter ces cartes ?

La réponse à cette question n'a été apportée que dans les deux dernières décennies. Il faut distinguer deux cas: les systèmes photoniques (nanoparticules métalliques supportant des plasmons, modes dans des guides d'ondes optiques, etc.) et les émetteurs semi-conducteurs (typiquement les matériaux III-N quantiquement confinés pour les technologies LEDs). Par

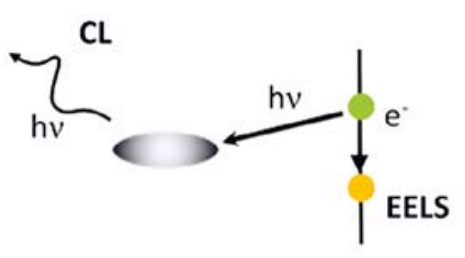

a

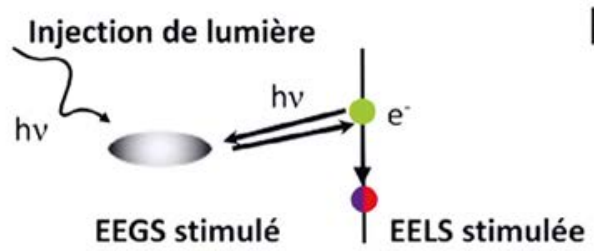

b

Figure 2.

Principe des spectroscopies de a. cathodoluminescence $(\mathrm{CL})$, de perte d'énergie électronique (EELS) et b. de gain d'énergie électronique (EEGS) et EELS stimulés.

exemple, dans le cas d'un nanoprisme d'argent, il parait évident que les excitations mises en jeu au sein du nanoprisme sont des plasmons de surface, les énergies mesurées en EELS et CL étant proches de celles mesurées en optique. Cependant, qualitativement, la question est plus délicate. En particulier, à quelle technique optique doit-on comparer les techniques de spectroscopie électroniques, et à quoi correspondent les variations spatiales des signatures spectroscopiques ? Comme souligné dans l'encart, on peut décrire, dans le cas de plasmons, l'EELS et la cathodoluminescence comme étant les contreparties nanométriques des spectroscopies d'extinction et de diffusion [3]. On note que le poids de ces signatures varie avec la position du faisceau électronique, ce qui bien sûr ne peut pas être mesuré avec des spectroscopies optiques conventionnelles qui sont limitées par la diffraction. Il faut alors plutôt comparer EELS et CL avec les techniques d'imagerie optique à sonde locale, telle que la microscopie de champ proche optique à balayage. Ces techniques mesurent plus généralement la densité d'états électromagnétique locale (EMLDOS). Cette quantité universelle quantifie les variations spectrales et spatiales du module du champs électrique associé à une nanoparticule ou plus généralementà tout objet perturbantlocalement le champ électromagnétique. On peut montrer quà peu de choses près, l'EELS mesure également directement l'EMLDOS, alors que la CL n'en mesure qu'une partie seulement (la partie radiative). Cela veut donc dire que l'EELS et la CL sont d'excellents outils pour sonder les propriétés optiques nanométriques de tous systèmes photoniques (plasmons, modes guidés, modes de galerie...) via leur EMLDOS.

En ce qui concerne les systèmes semi-conducteurs, l'EELS permet de mesurer la constante diélectrique locale, et permet particulièrement de

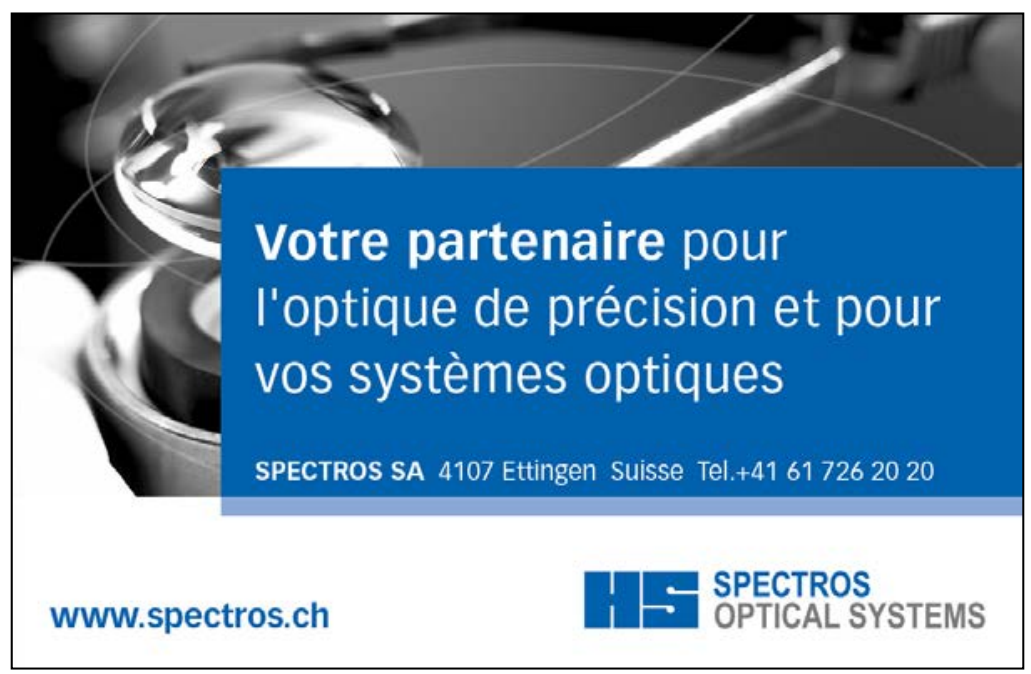


mesurer les valeurs des bandes d'énergieinterdites locales. La CL, quantà elle, permet essentiellement de retrouver le signal de luminescence qui aurait été généré par un rayonnement de haute énergie (c'est-à-dire, plus haut que la bande d'énergie interdite) en interaction avec les excitons et/ou les paires électrons-trous du matériau [2].

Ces problèmes conceptuels n'ontété soulevés, puis résolus, que très récemment, suivant l'avènement de nouvelles technologies instrumentales. En effet, l'EELS a souffert pendant longtemps de trois grands problèmes. Le premier était l'impossibilité d’obtenir une résolution spectrale inférieure à quelques centaines de $\mathrm{meV}$, voire $1 \mathrm{eV}$ (une précision médiocre quand on sait que le spectre visible sétend sur seulement environ 1,5 eV !) avec des résolutions nanométriques et un courant suffisant. Le second était l'impossibilité d'obtenir des informations à des énergies inférieures à $\sim 3 \mathrm{eV}$ (typiquement pour des longueurs d'onde inférieures au bleu). Ces deux problèmes ont été résolus avec l'introduction de nouvelles générations de monochromateurs (voir Fig. 1) permettant d'obtenir des résolutions aussi bonnes que $5 \mathrm{meV}$ et d'atteindre des domaines spectraux aussi bas que $40 \mathrm{meV}$. Le troisième problème résidait dans l'absence de détecteurs faiblement bruités, problème en partie résolu dans les années 2000 par l'introduction des caméras CCD, et maintenant complètement avec les caméras à détection directe délectrons. De manière similaire, la CL a été longtemps limitée par l'absence de détecteurs parallèles efficaces (CCD) ainsi que de techniques d'usinage des miroirs (voir Fig. 1) qui doivent être tout à la fois très fins (de 1 à qqes mm seulement) et très courbes [2]. Finalement, la spectroscopie de gain a grandement bénéficié de l'explosion des technologies laser femtosecondes à la base des microscopes électroniques ultra-rapides, dans lesquels le signal EEGS est très intense (voir article de A. Arbouet dans ce numéro).

\section{APPLICATIONS}

De nombreuses spectromicroscopies existent pour létude des phénomènes nanooptiques, parmi lesquelles on peut citer les techniques de super-résolutions tel le STED, si important pour les applications en bio-imagerie, ou les techniques de types SNOM ou de luminescence en STM, dont certaines permettent d'obtenir de l'imagerie vibrationnelles à l'échelle d'une molécule unique. Par rapport à ces techniques, les techniques S(T)EM se distinguent d’une façon générale par leur capacitéà étudier des échantillons relativement épais ainsi que la possibilité de corréler les informations optiques à des informations structurales, chimiques et électroniques éventuellement avec des résolutions atomiques. De plus, l'EELS possède également une dynamique spectrale inégalée (allant, dans un même instrument, de quelques dizaines de meV à des $\mathrm{keV}$ ).

Une large part des applications de l'EELS concerne la cartographie de plasmons, que nous avons déjà mentionnée, et d'autres excitations photoniques. Récemment, létude des excitations dans l'infra-rouge est devenue possible. On peut citer l'observation du couplage fort entre plasmons et phonons [4], ainsi que la cartographie de phonons à une échelle maintenant atomique [5] (Fig. 3). La CL, quant à elle, est particulièrement intéressante pour cartographier la luminescence, les recombinaisons de porteurs de charges dans des émetteurs quantiques [2] (Fig. 4) et de comparer quantitativement l'énergie d'émission à la taille des émetteurs, mesurées avec une
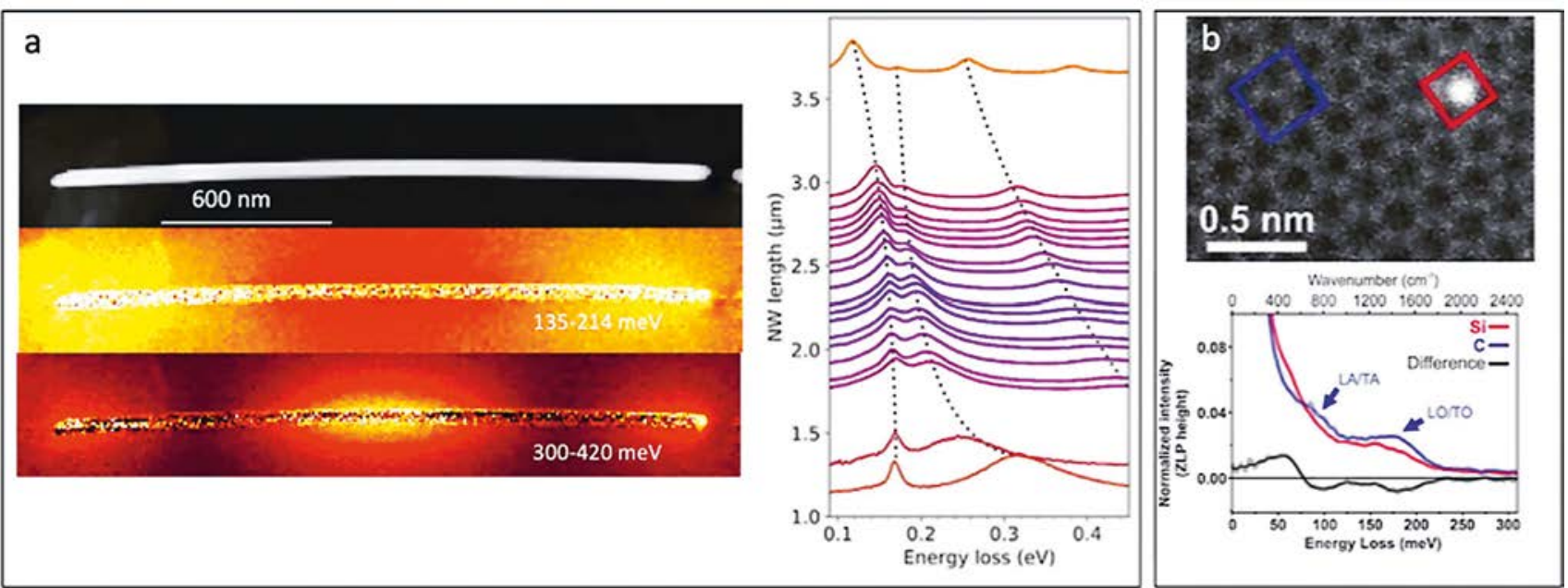

Figure 3.

Exemples récents d'applications en EELS. a. Spectroscopie EELS du couplage fort entre plasmons et phonons. A gauche, fond noir (haut) et images filtrées des modes dipolaires et quadrupolaires d'un nanofil d’argent sur du nitrure de bore (hBN). A droite: spectre EELS acquis à la pointe d'un nanofil (NW) dont la longueur est modifiée par découpe à l'aide du faisceau d'électron. On voit que le mode plasmon dipolaire se couple au phonon du hBN (vers 170 meV) pour des longueurs précises [4]. b. Spectroscopie EELS de vibration d’atomes uniques de silicium dans un plan de graphène. En haut : image de fond noir (l'atome de silicium apparaît plus clair). En bas, spectres EELS où l'on voit la signature des modes longitudinaux et transverses optiques et acoustiques (LO/TO/LA/TA). D'après [5]. 

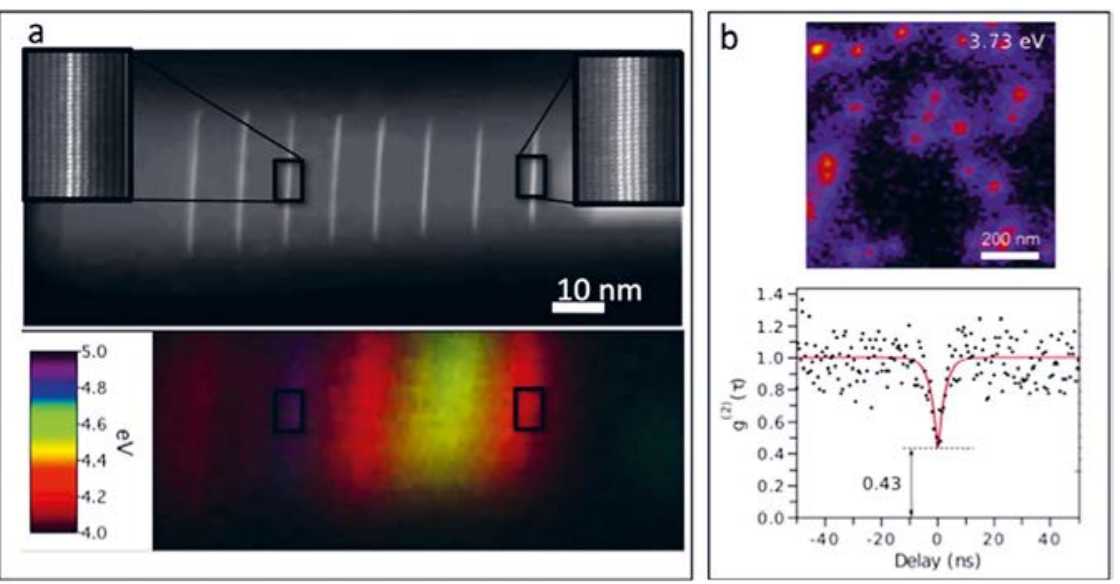

Figure 4.

Applications récentes de la $\mathrm{CL}$. a. Imagerie spectrale de puits quantiques confinés de GaN dans un fil d'AlN. En haut: image de fond noir. En insert, on discerne les plans d'atomes de Gallium (en blanc) permettant de mesurer l'épaisseur des puits. En bas: Image en fausses couleurs reconstituées à partir d'un spectre-image, permettant d'évaluer les variations d'énergie d'émission des puits en fonction de leur épaisseur mesurée sur le fond noir. b. Détection d'émetteurs de photons uniques dans du hBN. En haut: image filtrée à l'énergie d'un défaut ponctuel. En bas : fonction d’auto-corrélation du signal de CL présentant la signature d'un antiregroupement de photons caractéristique d'un émetteur de photons uniques. D’après [6].

précision atomique. Finalement, cette technique, connue pour être l'une des plus sensible pour la détection de défauts uniques, permet également de déterminer leur nature d'émetteurs de photon unique, par la mesure du phénomène d'anti-groupement des photons, un effet purement quantique [6]. Les techniques EEGS, plus récentes, commencent à peine à trouver leurs premières applications en nanooptique, comme par exemple la mesure de temps de vie d'excitations photoniques.

\section{CONCLUSION}

Les spectromicroscopies à base d'électrons rapides, de par leur résolution spatiale et spectrale, leur accès à de vastes domaines d'énergie et la possibilité de corréler ces informations à des informations structurales ou morphologiques se sont récemment imposées dans le domaine de la nanooptique. Il reste cependant de nombreux domaines où elles restent en retrait par rapport aux techniques purement optiques, comme par exemple la mesure de la polarisation ou le contrôle cohérent d'excitations (nano-) optiques. Il s'agit là de champs qui sont actuellement activement étudiés, et on peut espérer que les spectromicroscopies d'électrons rapides continueront à surprendre et exciter l'intérêt de la communauté scientifique dans les prochaines années.

\section{RÉFÉRENCES}

[1] F. Garcia de Abajo, Rev. Mod. Phys. 82, 209 (2010)

[2] M. Kociak, L. Zagonel, Ultramicroscopy 174, 50 (2017)

[3] M.Kociak, O. Stéphan, Chem. Soc. Rev. 43, 3865 (2014)

[4] L. Tizei et al., Nano Lett. 20, 2973 (2020)

[5] Hage et al., Science 367, 1124 (2020)

[6] R. Bourrellier et al., Nano Lett. 16, 4317 (2016)

L'OPTIQUE EST NOTRE AVENIR $^{\text {mm }}$

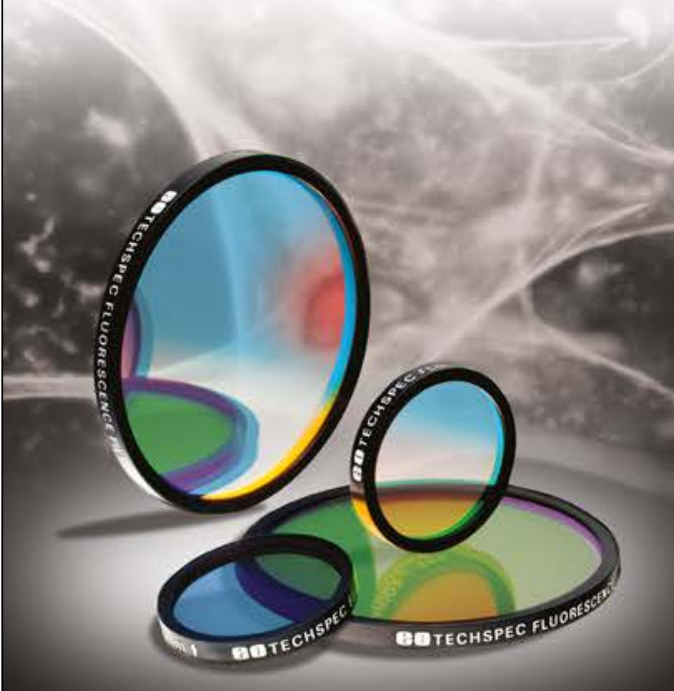

\section{TECHSPEC}

\section{Filtres Passe-Bande de Fluorescence}

- Traitement dur appliqué par pulvérisation, blocage OD 6

- Transmission > 93\% dans la bande passante

- Substrat de silice fondue de précision

Notre large gamme de plus de 65 filtres passe-bande de fluorescence a été spécialement conçue pour être utilisée avec les fluorophores courants. En plus de la microscopie à fluorescence, ces filtres sont des composants idéaux pour l'instrumentation spectroscopique, biotechnologique et de chimie clinique.

Pour en savoir plus :

\section{www.edmundoptics.fr/ fluorescence}

+33(0) 820207555 sales@edmundoptics.fr 